\title{
THz Generation using Nonlinear Optics: Mathematical Analysis and Design of THz Antennas
}

\author{
Manisha Khulbe1, Malay Ranjan Tripathy², Harish Parthasarathy³, \\ ${ }^{1}$ Department of Electronics and Communication Engineering, Ambedkar Institute of Advanced Communication Technologies \\ and Research, Delhi, India \\ ${ }^{2}$ Department of Electronics and Communication Engineering, Amity School of Engineering and Technology, Amity University \\ Noida, U.P, India \\ ${ }^{3}$ Department of Electronics and Communication Engineering, Netaji Subhash Institute of Technology, N. Delhi, India \\ E-mail: manisha.khulbe@gmail.com
}

\begin{abstract}
In this paper, a mathematical model is designed for $\mathrm{THz}$ generation from a nonlinear random medium. Using nonlinear optics and Maxwell's equations a set of nonlinear wave equations are derived to give scattered electromagnetic fields from an inhomogeneous medium. First, the analysis is done with second order nonlinearity. Its scattering parameters are calculated for $\mathrm{THz}$ radiation. Secondly, third order nonlinearity is described in terms of the coupling of Scalar and vector field components of nonlinear wave equations. The coupling of waves results in soliton generation. Multiple nonlinear interaction in the medium also gives wide bandwidth. Speed and high bandwidth is a demand of future networks. Hence a mathematical proof is implemented for $\mathrm{THz}$ antennas using SHG (Second harmonic generation) and THG (Third harmonic generation) materials. These antennas are designed and simulated using GaAs as a SHG material, and Graphene with $\mathrm{SiO} 2$ substrate as a THG material. GaAs is used as a substrate, which radiates at 524.8 $\mathrm{GHz}$ giving a high bandwidth of $25 \mathrm{GHz}$. Similarly, Graphene patch antenna with $\mathrm{SiO} 2$ substrate radiates in $\mathrm{THz}$ region at $3.5 \mathrm{THz}$ giving very high bandwidth of 2.5THz.Graphene patch antenna shows variation in radiation and bandwidth with the change of substrate of second order and third order nonlinear material. This change is observed with the antenna geometry. The difference between a bulk material antenna for $\mathrm{THz}$ generation and graphene antenna is elaborated. Graphene in terms of bandwidth and radiation can be better tuned with substrate material and geometry.
\end{abstract}

\subsection{Introduction}

$\mathrm{THz}$ technology is playing an important role in the area of sensing, imaging ${ }^{1}$ and wireless communication. This technology uses the spectrum falling between microwave and infrared radiation. It is called submillimeter radiation. These waves travel in line of sight and are nonionizing. Electromagnetic radiation from 0.3 to $10 \mathrm{THz}$ falls in the $\mathrm{THz}$ region. Although $\mathrm{THz}$ waves are highly penetrating, some limitations are also there. Earth's atmosphere is a strong absorber of $\mathrm{THz}$ radiation in specific water vapor absorption bands so the range of $\mathrm{THz}$ radiation is limited enough in longrange communication ${ }^{2}$. However, these waves are suitable for the communication range of near 10-meter specially imaging, high bandwidth wireless networking systems for indoor systems ${ }^{2}$.

In the generation of $\mathrm{THz}$ waves nonlinear optics plays a crucial role. $\mathrm{THz}$ generation from nonlinear optical response of free electrons in metals has been given by Zhang et al. (2016) based on SHG, which has been studied extensively in the past few decades. The typical fields are generated using $\mathrm{ZnTe}$ nonlinear crystals $^{3}$. The approximation of the nonlinear polarization of the metal surface is described by the susceptibility tensor $\chi_{\mathrm{ijk}}$ by solving the Maxwell's ${ }^{3}$ equations. Using nonlinear optical properties Nano antennas can be fabricated which are resonating devices, which convert EM waves into a localized energy in $\mathrm{THz}$ waves. The size and geometry of the antenna will control the antenna radiation $^{3}$.Some applications such as imaging, spectroscopy, and pharmaceutical application has been elaborated by Pawar and Zhang ${ }^{2,3}$.THz wave has both wave nature and particle nature. It has good penetration for some dielectric materials and nonpolar materials ${ }^{4}$.Penetrability makes it a supplement for $\mathrm{X}$ - ray imaging and ultrasound imaging ${ }^{4}$.THz radiation has a sufficiently small wavelength that it can discern small structures of millimeter size. It can therefore be used for a number of imaging applications, such as scanning of material defects, scanning of weapons, explosives and in the area of medical imaging $\mathbf{4 , 5}$.

In this paper, we derive a scattered electromagnetic wave equation from a nonlinear medium using nonlinear optics. Medium is assumed as isotropic and anisotropic ${ }^{6}$. Output wave is obtained by varying the susceptibility pattern of the medium. When a high intensity electromagnetic wave passes through these nonlinear medium, SHG (Second harmonic generation) and THG (Third harmonic generation) waves are generated in $\mathrm{THz}$ region. $\mathrm{THG}$ waves are generated by the Kerr nonlinearity. These nonlinear waves also produce solitons. It is also believed that stronger nonlinearities could 
be obtained at lower frequencies such as infrared and terahertz frequencies ${ }^{7}$.

Antenna using SHG is designed in HFSS with GaAs substrate. We achieve a high bandwidth antenna. Because of high bandwidth, THz is being used for short-range high-speed data networks ${ }^{5}$. Nonlinear wave equation using Kerr nonlinearity is derived which has scalar and vector wave components. If these two nonlinear wave equations are coupled then we achieve a high bandwidth by taking the Fourier transform of coupled wave equations. In addition, there exists number of modes for a bounded medium. This paper focuses on the $\mathrm{THz}$ generation from second harmonic and third harmonic materials. Kerr nonlinearity also known as third order nonlinearity also giving $\mathrm{THz}$ waves, which is being generated from the centrosymmetric materials ${ }^{6}$.Prominent feature of nonlinear medium is the generation of new frequencies. For cubic term in polarization functional, there is nonlinear contribution to the basic frequency, which is known as self-focusing effect. It has been reported by Zielinska J.A and Mitchell, M.W., 2017, see ref [8].There is Self-compression of high-intensity femtosecond/THz pulses using third order nonlinearity in some cases and solid materials ${ }^{9}$, which also gives spatiotemporal solitons, the combined action of selfcompression and self-focusing effect.

In this paper, section 2.1 gives mathematical detail description of the First order and second order harmonic wave, generation. In Section 2.2, third order Harmonic wave generation is discussed and different nonlinear material including SHG and THG are elaborated in 2.3. Section 3 describes the HFSS design of $\mathrm{THz}$ antenna taking GaAs substrate (SHG) and Graphene (THG) antenna with $\mathrm{SiO} 2$ substrate (THG). Details of experimental results and discussions are given in section 4.In section 5 difference between $\mathrm{THz}$ antennas using bulk material and $\mathrm{THz}$ antennas using nonlinear materials have been described. Section 6 gives useful applications of a nonlinear wave equation generating $\mathrm{THz}$ waves. Section 7 concludes the paper.

\subsection{Scattering from nonlinear random medium: mathematical analysis}

\subsection{Second order nonlinearity:}

The far field radiation generated given by a set of nonlinear wave equations assuming a nonlinear material, which will be probe fed from a high intensity wave. A receiver can also be designed using nonlinear wave optics. If a laser source is applied at the nonlinear crystal substrate, the crystal operates in linear and nonlinear region. The incident wave polarizes the material. The polarization depends on the material is isotropic or anisotropic. In the nonlinear region, the motion of electron is in the direction of polarization. Electromagnetic field is described by a second order nonlinear differential equation with a secondary source contributed by nonlinear polarization $\mathrm{P}^{\mathrm{NL}}$.

$$
\nabla \times \nabla \times E+\mu_{0} \epsilon \frac{\partial^{2} E}{\partial t^{2}}=-\mu_{0} \frac{\partial^{2} P^{N L}}{\partial t^{2}}
$$

This is an inhomogeneous wave equation where $\mathrm{P}^{\mathrm{NL}}$ is the nonlinear response drives of the electric field. Since $\frac{\partial^{2} P^{N L}}{\partial t^{2}}$ is the measure of acceleration of the charges that constitutes the medium, this equation is consistent with the Larmour's theorem of electromagnetism which states that accelerated charges generate electromagnetic radiation ${ }^{11}$. The time varying polarization can act as the source of new component of the electromagnetic fields. This contributes to the polarization $\mathrm{P}$ in the electric dipole moment per unit volume. Electric field generated by electric current source $J$ at a distance $r$ assumed to be produced by the random movement of dipoles is given by

$\mathrm{A}=\frac{\mu}{4 \pi} \iiint_{\mathrm{V}} \frac{\mathrm{J} \mathrm{e}^{-\mathrm{ikr}}}{\mathrm{r}} \mathrm{dV}$ which is a solution to the wave equation $^{12}$. The Helmholtz wave equation is given by-

$$
\nabla^{2} A+K^{2} A=\mu J
$$

Where $K$ is a propagation constant. If we take a small current because of a small dipole and the radiation field due to dipoles, travelling in the outward direction is followed by the equation, which is given by

$$
\nabla^{2} E+K^{2} E=0
$$

$\left(J_{Z}=0\right)$ (When the point source is removed)

Considering a dense pack of such dielectric sphere of dipoles of random size scattering classical waves. For example, point scatterer distributed statistically throughout random medium as described by the wave equation for the scalar field can be given by

$$
\left(c^{2}(r) \frac{\partial^{2}}{\partial t^{2}}-\nabla^{2}\right) \psi(r, t)=0
$$

Where $\psi(r, t)$ is defined as a wave function. Randomly varying phase velocity is $\frac{1}{\sqrt{\epsilon_{r} \mu_{r}}}$, we assume spatially varying Dielectric constant $\epsilon_{r}$.Also assume that the fluctuation of $\epsilon_{r}$ to spatially uncorrelated in order material to be random ${ }^{12}$.

$$
\epsilon_{r}=\bar{\epsilon} \text { And }<\epsilon_{1}(r) \epsilon_{2}(r)>=\delta\left(r-r^{\prime}\right)
$$

If the current densities in $\mathrm{x}$ and $\mathrm{y}$ directions are $\mathrm{J}_{\mathrm{x}}$ and $\mathrm{J}_{\mathrm{y}}$ the wave equations for each would reduce to

$$
\begin{gathered}
\nabla^{2} A_{x}+K^{2} A_{x}=\mu J_{x} \\
\nabla^{2} A_{y}+K^{2} A_{y}=\mu J_{y}
\end{gathered}
$$

With corresponding solution in the form of

$$
\begin{aligned}
& A_{x}=\frac{\mu}{4 \pi} \iiint_{V} \frac{J_{x} e^{-i k r}}{r} d V^{\prime} \\
& A_{y}=\frac{\mu}{4 \pi} \iiint_{V} \frac{J_{y} e^{-i k r}}{r} d V^{\prime}
\end{aligned}
$$

If the source is removed from the origin and placed at a position represented by the coordinate $\left(\mathrm{x}^{\prime}, \mathrm{y}^{\prime}, \mathrm{z}^{\prime}\right)$ it can be written as in terms of the retarded potential

$\mathrm{A}(\mathrm{x}, \mathrm{y}, \mathrm{z})=\frac{\mu}{4 \pi} \iiint_{V} \frac{J\left(x^{\prime}, y^{\prime}, z^{\prime}\right) e^{-i k|r-r \prime|}}{\left|r-r^{\prime}\right|} d V^{\prime}$

Electric field propagating in $\mathrm{z}$ direction is defined by-

$$
\mathrm{E}(\mathrm{z}, \mathrm{t})=\hat{a}_{x} E(z, t)+\hat{a}_{y} E(z, t)
$$




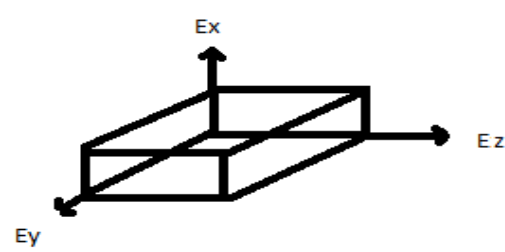

Figure 1: Scattering volume $\Delta V=\Delta x \Delta y \Delta z$

$$
\begin{gathered}
E_{x}(z, t)=\operatorname{Re}\left[E_{x 0} e^{i \omega\left(t+k z+\emptyset_{x}\right.}\right] \\
E_{y}(z, t)=\operatorname{Re}\left[\mathrm{E}_{\mathrm{y} 0} \mathrm{e}^{\mathrm{i} \omega\left(\mathrm{t}+\mathrm{kz}+\emptyset_{\mathrm{x}}\right.}\right]
\end{gathered}
$$

In far field region $E_{A}=-j \omega A$

Where $\quad A(x, y, z)=\hat{a}_{z} \frac{\mu_{0}}{4 \pi r} I e^{-i k r} \int_{-l / 2}^{l / 2} d z$

Hence incident field $\mathrm{E}$ is proportional to the $\mathrm{A}$ vector potential generated by an infinitesimal dipole in a volume $\Delta V=\Delta x \Delta y \Delta z$ Where $\quad \Delta x \approx r$ hence taking

$$
\hat{E}^{0}(\omega, r)=\frac{-j \omega \mu}{4 \pi} \iiint_{V} \frac{J(\omega, r) e^{-i k|r-r \prime|}}{\left|r-r^{\prime}\right|} d^{3} r
$$

Which is a solution to the wave equation

$$
\nabla^{2} E^{0}+K^{2} E^{0}=j \omega \mu \tilde{J}
$$

Thus, fundamental wave is defined by the equation 15 .

The nonlinear relationship between the existing electric field $\mathrm{E}$ and resulting polarization $\mathrm{P}$ for weak excitation is linear and most optical antenna operate in this region however for strong excitation the response $\mathrm{P}$ depends on higher powers of $\mathrm{E},{ }^{14}$. If $\mathrm{E}$ has perturbation term then

$$
E=\left(E^{0}(\omega, r)+\delta E^{1}(\omega, r)+\delta^{2} E^{2}(\omega, r)+\cdots\right)
$$

Using equation 15 in the Polarization term ${ }^{6,10}$

$P^{N L}=\epsilon_{0}\left(\chi^{1} \hat{E}+\chi^{2}: \hat{E} \otimes \hat{E}+\chi^{3}: \hat{E} \otimes \hat{E} \otimes \hat{E}+\ldots\right)$

$\left[\chi^{1} \hat{E}=\chi^{1}(\omega, r) \hat{E}(\omega, r)\right.$

$$
\begin{gathered}
\chi^{2}: \hat{E} \otimes \hat{E}=\left(\chi^{2}: \hat{E} \otimes \widehat{E}\right)(\omega, r) \\
=\int \chi^{2}\left(\omega^{\prime}, \omega-\omega^{\prime}, r\right)\left(\hat{E}\left(\omega^{\prime}, r\right) \otimes \hat{E}(\omega-\right. \\
\left.\left.\left.\omega^{\prime}, r\right)\right) \mathrm{~d} \omega^{\prime}\right]
\end{gathered}
$$

These expressions are derived by Fourier transforming the corresponding time domain expressions, which in turn may be derived by looking at the motion of an electron bound to the nucleolus by an harmonic potential and subjected to an external varying electric field and then conducting its dipole moments, based on perturbation theory for ordinary differential equations.

We consider a relation between polarization and electric field as $\mathbf{1 5 , 1 6}$

$$
\begin{aligned}
& \quad\left\{P_{i}\right\}(\mathrm{r}, \mathrm{t})=\varepsilon_{0}\left\{\int d t_{1} \chi^{1}{ }_{i j}\left(t_{1}\right) E_{j}\left(r, t-t_{1}\right)+\right. \\
& \int d t_{1} \int d t_{2} \chi^{2}{ }_{i j k}\left(t_{1}, t_{2}\right) E_{j}\left(r, t-t_{1}\right) \otimes E_{k}(r, t- \\
& \left.t_{2}\right)+\int d t_{1} \int d t_{2} \chi^{3}{ }_{i j k}\left(t_{1}, t_{2}, t_{3}\right) E_{j}(r, t- \\
& \left.\left.t_{1}\right) \otimes E_{k}\left(r, t-t_{2}\right) \otimes E_{l}\left(r, t-t_{3}\right)\right\}
\end{aligned}
$$

The equation also defines the anisotropicity when nth order polarization is taken:

$$
\left\{P_{i}\right\}^{n}=\varepsilon_{0} \int d t_{1} \ldots \int d t_{n} \cdot \chi^{n}{ }_{i p_{1} \ldots p_{n}}{ }^{\left(t_{1} \ldots, t_{n}\right) E_{p_{1}}\left(r, t-t_{1}\right) \otimes \ldots \ldots E_{p_{n}}\left(r, t-t_{n}\right)}
$$

Where polarization constitutes a secondary source current inserted into Maxwell's equations giving rise to a set of nonlinear differential equations ${ }^{14}$.Using Maxwell's equations for an inhomogeneous, anisotropic medium, which may be defined in terms a random medium. Again, in terms of the time varying electric and magnetic fields the Maxwell's equations can be written in the form where $\mathrm{H}$ is the magnetic field component. Here we assume a nonmagnetic material.

$$
\begin{aligned}
& \nabla \times E=-j \omega \mu H \\
& \nabla \times H+\frac{\partial D}{\partial t} \\
& \nabla \times(\nabla \times E)=\nabla \times(-j \omega \mu(\vec{H}) \\
& \nabla^{2} E+\omega^{2} \mu_{0} \epsilon E-\nabla(\nabla . E)=\mathrm{j} \omega \mu_{0} \hat{J} \\
& \left(\nabla \times \nabla \times E=\nabla(\nabla . \mathrm{E})-\nabla^{2} E\right)
\end{aligned}
$$

Solution to this equation is obtained by adding perturbation to the higher order harmonics of the electric field in terms of Taylor series expansion. First order electric field is calculated in terms of fundamental wave. Similarly second order perturbation in electric field is calculated in terms of fundamental wave and first order wave. Set of homogeneous and nonhomogeneous equations are obtained by solving the nonlinear wave equation 22 .

Substituting in equation $22 \epsilon E=\varepsilon_{0} E+P$

$$
\begin{aligned}
& \nabla^{2}\left(E^{0}(\omega, r)+\delta E^{1}(\omega, r)+\delta^{2} E^{2}(\omega, r)+\cdots\right)+ \\
& \omega^{2} \mu_{0} \epsilon_{0}\left(E^{0}(\omega, r)+\delta E^{1}(\omega, r)+\delta^{2} E^{2}(\omega, r)+P\right) \\
& \nabla\left(\nabla .\left(E^{0}(\omega, r)+\delta E^{1}(\omega, r)+\delta^{2} E^{2}(\omega, r)+\cdots\right)\right)=\mathrm{j} \omega \mu_{0} \hat{J}
\end{aligned}
$$

$P^{N L}=\delta \epsilon_{0} \chi^{1}+\delta^{2} \epsilon_{0} \chi^{2}: \hat{E} \otimes \hat{E}+\delta^{3} \epsilon_{0} \chi^{3}: \hat{E} \otimes \widehat{E} \otimes \hat{E} \quad 10$

Substituting equations 17,18 and 25 in equation 22 we get $\nabla^{2}\left(E^{0}(\omega, r)+\delta E^{1}(\omega, r)+\delta^{2} E^{2}(\omega, r)+\cdots.\right)+$ $\omega^{2} \mu_{0} \epsilon_{0}\left(E^{0}(\omega, r)+\delta E^{1}(\omega, r)+\delta^{2} E^{2}(\omega, r)+\right.$ $\delta \chi^{1}\left(E^{0}(\omega, r)+\delta E^{1}(\omega, r)+\delta^{2} E^{2}(\omega, r)\right)+$ $\delta^{2} \chi^{2}:\left(E^{0}(\omega, r)+\delta E^{1}(\omega, r)+\right.$ $\left.\delta^{2} E^{2}(\omega, r)\right) \otimes\left(E^{0}(\omega, r)+\delta E^{1}(\omega, r)+\delta^{2} E^{2}(\omega, r)\right)+$ $\delta^{3} \chi^{3}:\left(E^{0}(\omega, r)+\delta E^{1}(\omega, r)+\right.$ $\left.\delta^{2} E^{2}(\omega, r)\right) \otimes\left(E^{0}(\omega, r)+\delta E^{1}(\omega, r)+\right.$ $\left.\left.\delta^{2} E^{2}(\omega, r)\right) \otimes\left(E^{0}(\omega, r)+\delta E^{1}(\omega, r)+\delta^{2} E^{2}(\omega, r)\right)\right) \quad-$ $\nabla\left(\nabla .\left(E^{0}(\omega, r)+\delta E^{1}(\omega, r)+\delta^{2} E^{2}(\omega, r)+\cdots \cdot\right)\right)=\mathrm{j} \omega \mu_{0} \hat{\jmath}$

Comparing the powers of $\delta$ both sides in equation 26, we get the Helmholtz equation for fundamental wave

$$
\nabla^{2} E^{0}+K^{2} E^{0}=0
$$

From Maxwell's equation $-\operatorname{div}(\mathrm{D})=0$ 


$$
\operatorname{div}\left(\varepsilon_{0} E+P\right)=0
$$

If perturbation is added to each nonlinear term thenD. $\left(\varepsilon_{0} \hat{E}+\epsilon_{0}\left(\delta \chi^{1} \hat{E}+\delta^{2} \chi^{2}: \widehat{E} \otimes \hat{E}+\right.\right.$

$\left.\left.\delta^{3} \chi^{3}: \hat{E} \otimes \hat{E} \otimes \hat{E}\right)\right)=0$

From equation, 17 put the values of $\mathrm{E}$ in equation 28 we get-

$$
\begin{array}{lc}
\delta^{0} & \nabla \cdot\left(\varepsilon_{0} E^{0}\right)=0 \\
\delta^{1}: & \nabla \cdot \varepsilon_{0}\left(E^{1}\right)+\varepsilon_{0} \nabla \cdot\left(\chi^{1} E^{0}\right)=0 \\
& \nabla \cdot\left(E^{1}\right)=-\nabla \cdot\left(\chi^{1} E^{0}\right) \\
\delta^{2}: \nabla . \varepsilon_{0}\left(E^{2}\right)+\varepsilon_{0} \nabla \cdot\left(\chi^{1} E^{1}+\chi^{2} E^{0} \otimes E^{0}\right)=0
\end{array}
$$

Now comparing both LHS and RHS powers of $\delta^{0}, \delta^{1}, \delta^{2}$ from equation from equation 24 we get the solutions of the scattered waves-

$$
\begin{aligned}
& \nabla^{2}\left(E^{0}(\omega, r)+\omega^{2} \mu_{0} \epsilon_{0}\left(E^{0}(\omega, r)-\right.\right. \\
& \nabla\left(\nabla .\left(E^{0}(\omega, r)\right)=\mathrm{j} \omega \mu_{0} \hat{J}\right.
\end{aligned}
$$

From equation 31

$$
\begin{gathered}
\nabla \cdot\left(\varepsilon_{0} E^{0}\right)=0 \\
\nabla^{2}\left(E^{0}(\omega, r)+\omega^{2} \mu_{0} \epsilon_{0}\left(E^{0}(\omega, r)\right)=\mathrm{j} \omega \mu_{0} \hat{J}\right.
\end{gathered}
$$

Using Green's function, we can find out $E^{0}$

$$
\begin{array}{r}
\nabla^{2} E^{0}+K^{2} E^{0}=\mathrm{j} \omega \mu \hat{\jmath},\left(\omega^{2} \mu_{0} \epsilon_{0}=K^{2}\right) \\
\left(\nabla^{2}+K^{2}\right) E^{0}=\mathrm{j} \omega \mu \hat{\jmath}
\end{array}
$$

Using Green's function -

$$
E^{0}=\left(\frac{-1}{4 \pi}\right) \int_{0}^{1} \frac{\mathrm{j} \omega \mu \hat{J}}{|r-r \prime|} e^{-i k\left(r-r^{\prime}\right)} d^{3} r^{\prime}
$$

Comparing the $\delta^{1}$ terms:

$\nabla^{2}\left(E^{1}(\omega, r)\right)+\omega^{2} \mu_{0} \epsilon_{0} E^{1}(\omega, r)+\epsilon_{0} \chi^{1} E^{0}-$ $\nabla\left(\nabla .\left(E^{1}(\omega, r)\right)=0\right.$

$\nabla^{2}\left(E^{1}(\omega, r)\right)+\omega^{2} \mu_{0} \epsilon_{0} E^{1}(\omega, r)+$

$K^{2} \chi^{1} E^{0}(\omega, r)+\nabla\left(\nabla \cdot \chi^{1} E^{0}(\omega, r)\right)=0$

Using Green's function

$$
\begin{aligned}
& E^{1}(\omega, r)= \\
& \left(\frac{-1}{4 \pi}\right) \int_{0}^{1} \frac{K^{2} \chi^{1} E^{0}\left(\omega, r^{\prime}\right)+\nabla\left(\nabla \cdot \chi^{1} E^{0}\left(\omega, r^{\prime}\right)\right)}{\left|r-r^{\prime}\right|} e^{-i k\left|r-r^{\prime}\right|} d^{3} r^{\prime} \\
& \text { Comparing the terms we get- } \\
& \nabla^{2} E^{2}(\omega, r)+K^{2} E^{2}(\omega, r)+K^{2}\left(\chi^{1} E^{1}+\right. \\
& \left.\chi^{2} E^{0}(\omega, r) \otimes E^{0}(\omega, r)\right)-\nabla\left(.\left(\nabla \cdot \left(\chi^{1} E^{1}+\right.\right.\right. \\
& \left.\left.\chi^{2}\left(E^{0} \otimes E^{0}\right)\right)=0\right) \\
& \left(\nabla^{2}+K^{2}\right) E^{2}(\omega, r)=-\left(K ^ { 2 } \left(\chi^{1} E^{1}+\right.\right. \\
& \left.\left.\left.\chi^{2} E^{0}(\omega, r) \otimes E^{0}(\omega, r)\right)\right)+\nabla\left(\nabla \cdot\left(\chi^{1} E^{1}+\chi^{2} E^{0} \otimes E^{0}\right)\right)\right) \\
& \left.\left.\int_{0}^{1} \frac{E^{2}(\omega, r)=\left(\frac{-1}{4 \pi}\right) .}{\nabla\left(\nabla \cdot \chi^{2} E^{0}\left(\omega, r^{\prime}\right) \otimes E^{0}\left(\omega, r^{\prime}\right)\right.}\right)\right) \\
& \left|r-\gamma^{\prime}\right| \\
& .\left(K^{2}\left(\chi^{1} E^{1}\left(\omega, r^{\prime}\right)+\chi^{2} E^{0}(\omega, r) \otimes E^{0}\left(\omega, r^{\prime}\right)\right)\right)+ \\
& \int^{-i k\left|r-r^{\prime}\right|} d^{3} r^{\prime}
\end{aligned}
$$

Equation 39 is the first order scattered waves .Equation 42 is the Second Harmonic wave. $\chi^{1}$ and $\chi^{2}$ are the linear and nonlinear susceptibility tensors of the medium. $\chi^{1}$ is a $3 \times 3$ matrix valued function of $(\omega, \mathrm{r})$ while $\chi^{2}$ is a $3 \times 6$ matrix valued function of $(\omega, r)$. The second order nonlinear polarization $\mathrm{P}_{2 \omega}(\mathrm{t})^{17}$ in terms of frequency is given by-

$$
\begin{gathered}
P_{2 \omega}(t)= \\
\epsilon_{0}\left(\begin{array}{llllll}
0 & 0 & 0 & d & 0 & 0 \\
0 & 0 & 0 & 0 & d & 0 \\
0 & 0 & 0 & 0 & 0 & d
\end{array}\right)\left(\begin{array}{c}
E_{\omega, x}{ }^{2}(t) \\
E_{\omega, y}{ }^{2}(t) \\
E_{\omega, z}{ }^{2}(t) \\
2 E_{\omega, y}(t) E_{\omega, z}(t) \\
2 E_{\omega, z}(t) E_{\omega, x}(t) \\
2 E_{\omega, x}(t) E_{\omega, y}(t)
\end{array}\right)
\end{gathered}
$$

$E_{\omega}(t)$ is the electric field amplitude of the nearly monochromatic fundamental wave $\mathrm{E}_{\omega}(\mathrm{t}) \mathrm{e}^{-\mathrm{ikr}-\mathrm{j} \omega \mathrm{t}}$. The subscript $\mathrm{x}, \mathrm{y}, \mathrm{z}$ represent the crystal directions $\left(\begin{array}{lll}1 & 0 & 0\end{array}\right),\left(\begin{array}{ll}0 & 1\end{array}\right.$ $0)$ and $\left(\begin{array}{lll}0 & 0 & 1\end{array}\right)$.The second order nonlinear susceptibility is found in $\mathrm{ZnTe}, \mathrm{ZnSe}$ and $\mathrm{ZnS}{ }^{16}$. For second order nonlinearity can also be defined in terms of first order nonlinearity ${ }^{15} \cdot \chi^{2}\left(\mathrm{t}_{1}, \mathrm{t}_{2}\right)=-\mathrm{k} \varepsilon_{0}{ }^{3} \int \chi^{1}\left(\mathrm{t}^{\prime}\right) \chi^{1}\left(\mathrm{t}_{1}-\right.$

$\left.t^{\prime}\right) \chi^{1}\left(t_{2}-t^{\prime}\right) d t^{\prime}$.There also exists a linear bound between second order polarization and first order polarization and it is also invariant under time translation. It exists for all higher polarizations.

\subsection{Third order nonlinearity (Kerr nonlinearity):}

Similar to second order nonlinearity we assume a volume of $\Delta x \Delta y \Delta z=\Delta v$ of a nonlinear crystal which is being probe fed by a laser. In third order, nonlinearity the refractive index of the material depends on the electromagnetic energy, known as a Kerr nonlinearity. The nonlinear effect of molecular direction is an anisotropic effect ${ }^{\mathbf{1 5}}$. When this volume is probe fed by a laser this will acts like a cavity resonator converting to a frequency, which is produced by the coupling of nonlinear waves inside the cavity. The cavity is also compared to a Fabry Perot resonator. Hence, it is a selfstabilized cavity resonated optical frequency converter using Kerr nonlinearity.

2.2.1 Mathematical analysis: If there is electric field E, magnetic field $\mathrm{H}$ and susceptibility variation with respect to $\mathrm{E}$ and $\mathrm{H}$ which is also known as kerr nonlinearity. The susceptibility is varying with respect to electric field and permeability is varying by magnetic field produced by the electromagnetic field.

Using Maxwell's equations-

$$
\begin{aligned}
& \operatorname{div}(\epsilon E)=0 \\
& (\nabla \epsilon, E)+\epsilon \operatorname{div} \mathrm{E}=0,
\end{aligned}
$$

If change in the susceptibility is defined by the following equations ${ }^{18}$ which is defined by electric and magnetic susceptibilities. It can be written in terms of $i, j, k$ indices as variation of susceptibility will be in three-dimensional plane. 
$\nabla \chi_{e}=\frac{\partial \chi}{\partial E_{i}} \nabla E_{i}+\frac{\partial \chi}{\partial E_{j}} \nabla E_{j}+\frac{\partial \chi}{\partial E_{k}} \nabla E_{k} ; \nabla \chi_{m}=\frac{\partial \chi}{\partial H i} \nabla H_{i}+$

$\frac{\partial \chi}{\partial H j} \nabla H_{j}+\frac{\partial \chi}{\partial H k} \nabla H_{k}$

$\nabla \chi_{e}=\sum_{i, j=1}^{3} E_{x} \frac{\partial \chi}{\partial E_{j}} E_{j i} \widehat{e}_{l}$ Where $E_{j i}=\frac{\partial E_{j}}{\partial X_{i}}$

Then Maxwell's equation 21 can be written as

$\nabla \times(\nabla \times E)=-\nabla \times\left(\frac{\partial}{\partial t} \mu(\vec{H}) \vec{H}\right)$

Expanding equation $48^{18}$ -

$\nabla \operatorname{div} E-\nabla^{2} E=-\nabla \frac{\partial(\mu \times \mathrm{H})}{\partial \mathrm{t}}-\nabla \mu \times \frac{\partial H}{\partial t}-\frac{\partial \mu(\nabla \times \mathrm{H})}{\partial \mathrm{t}}-\frac{\mu \partial(\nabla \times \mathrm{H})}{\partial \mathrm{t}}$

From equations 45 and 47 we can write equation 50 where $\alpha_{i j}$ is the kerr coefficient for the medium-

$\sum_{i, j=1}^{3}\left(\alpha_{i j} \frac{\partial \chi}{\partial E_{j}} E_{j i} E_{k} \cdot \widehat{e_{j}}\right) E_{k}=M^{\prime}$

$\nabla^{2} \mathrm{E}+\nabla \cdot \mathrm{M}^{\prime}=-\nabla \frac{\partial(\mu \times \mathrm{H})}{\partial \mathrm{t}}-\nabla \mu \times \frac{\partial H}{\partial t}-\partial \frac{\mu(\nabla \times \mathrm{H})}{\partial \mathrm{t}}-\frac{\mu \partial(\nabla \times \mathrm{H})}{\partial \mathrm{t}}$

Permeability

$\mu_{0} \nabla \chi_{\mathrm{m}}(\mathrm{H})$, where $\nabla \mu_{0}=0$ $\mu=\mu_{0}\left(1+\chi_{m} H\right), \nabla \mu=$

Magnetic permeability variation with magnetic field is given in terms of $i^{\prime} j^{\prime} k^{\prime}$ indices as ${ }^{18}$

$$
\nabla \chi_{m}=\mu_{0} \sum_{\substack{i^{\prime}, j^{\prime} \\=1}}^{3}\left(\alpha_{i^{\prime} j^{\prime}} \frac{\partial \chi}{\partial H_{y}} H_{j, i^{\prime}} H_{j^{\prime}}, \widehat{e_{J^{\prime}}} H_{k^{\prime}}\right)
$$

Hence using equation 50 and 52 in equation 51 , we get the final nonlinear wave equation, which is having scalar and vector wave components. Kavitha, etal (2016) [20] proved the coupling of magnetic and electric field vectors and produced soliton in a antiferromagnetic medium.

There is slowly varying amplitude approximations of the output of nonlinear waves, which is approximated to soliton generation in the lossless medium ${ }^{16}$. The approximations also leads to nonlinear Schrodinger equation ${ }^{15}$ for the envelope as discussed in section 4.0. Third order nonlinearity in isotropic case can also be given in terms of first order susceptibility variation $\chi^{1}{ }^{15} \chi^{3}\left(t_{1}, t_{2}, t_{3}\right)=-k$ $\varepsilon_{0}^{3} \int \chi^{1}\left(t^{\prime}\right) \chi^{1}\left(t_{1}-t^{\prime}\right) \chi^{1}\left(t_{2}-t^{\prime}\right) \chi^{1}\left(t_{3}-t^{\prime}\right) d t^{\prime}$.In the experiment done with equation 42 different noncentrosymmetic materials susceptibilities are given as input that shows output $\mathrm{THz}$ radiation giving different spectral peaks. In an experiment done by Ahmad etal 2013 see ref 21, with the Erbium-doped fiber laser (EDFL) which is a highly nonlinear fiber generates a stable soliton pulse train of 0.81 ps duration with repetition rate of $44 \mathrm{MHz}$ with average output power $92.4 \mu \mathrm{W}$ at a $980 \mathrm{~nm}$ pump power of $26.8 \mathrm{~mW}$.

\subsection{Nonlinear materials}

The second order nonlinearity is the lowest order nonlinearity and the first to be observed as intensity increases ${ }^{22}$.Materials without inversion symmetry are second order nonlinear material which are generally crystalline in nature. The SHG generation is due to this noncentrosymmetricity. This noncentrosymmetricity is found in many compounds like tubulin, muscle myosin found in human and animal biological cells .Because of this property of SHG, generation from noncentrosymmetric materials Biosensors are being developed ${ }^{23}$ which can also produce a terahertz wave at the output. Some other materials $\mathrm{ZnTe}, \mathrm{ZnSe}, \mathrm{LiNbO} 3$ are also SHG / noncentrosymmetric materials. $\mathrm{LiNbO3}$ is an anisotropic material, which has a high nonlinear coefficient and is widely being used in industry ${ }^{17}$.The experiments on second harmonic generation in II-VI semiconductor date back to the year 1963. Due to the limitation in the tunable high intensity lasers, the experiments were performed on limited crystals such as CdS, KDP. Second order nonlinear susceptibility in ZnTe, InSb, GaAs and InAs were carried out by Chang et al., (1965), Ref .24.These materials possess high SHG coefficients and thus have the potential for efficient SHG in the visible spectral region. Wide gap zinc blend II-VI semiconductor are optically isotropic but they do not possess a center of inversion ${ }^{24} \mathrm{ZnTe}$ and $\mathrm{ZnSe}$ are the SHG materials, which gives a $\mathrm{THz}$ response in the range 0.3 to 5 $\mathrm{THz}^{26}$.

Third order nonlinearity is found in centrosymmetric materials, which is also lowest order of nonlinearity ${ }^{15}$.Farazi and Zarifkar, 2017 in 27 have implemented a SHG and XPM (cross phase modulation) based Nano switch using centrosymmetric metals .SHG from gold is inspected and implemented for plasmonic switch.

One of the new material found is Graphene, which shows strong nonlinear behavior at microwave and terahertz frequencies ${ }^{7}$.It shows strong third order nonlinear effects due to inversion symmetry ${ }^{26}$. However at the interface of substrate and patch by breaking the symmetry it also behaves in the second order nonlinearity. This material shows remarkable optoelectronic properties with ultrafast response time and broad spectral range. The susceptibility value of monolayer Graphene is eight orders of magnitude higher than the same nonlinearity in normal dielectrics ${ }^{28}$. The large nonlinearity of Graphene is also used as a tool in FWM (four wave mixing) imaging. Graphene also shows $\mathrm{THz}$ electromagnetic wave radiation ${ }^{28}$.

\subsection{THz Antennas (Experiments in HFSS)}

3.1. Using a second harmonic generation material (GaAs) as a substrate a patch antenna is designed in HFSS. It verifies the scattering of electromagnetic waves from these materials when electromagnetic incident wave is incident on it. This antenna is designed with a geometry of $\mathrm{T}$ shape patch at the four corners with a square patch at the center shown in section 4.0. GaAs is used, as a nonlinear substrate .It is probe fed at two points ${ }^{29}$. Experiments were carried out using finite element method in HFSS. This experiment proves that there 
is high frequency emission of $\mathrm{THz}$ waves from the $\mathrm{SHG}$ material. It also gives wide bandwidth in $\mathrm{GHz}$ range.

3.2. Another experiment was carried out with antenna designed using Graphene patch over a nonlinear substrate of $\mathrm{SiO} 2$ a third order nonlinear material ${ }^{28}$.The antenna is designed with a bow tie geometry. As Graphene with a loss tangent 0.05 and relative permittivity 3.2 is a highly optical nonlinear material. It radiates in $\mathrm{THz}$ when feeded by an electromagnetic wave at the center of the bow tie geometry. The radiation and Gain curves for this $\mathrm{THz}$ antenna are shown in 4.0. These results prove the scattering of electromagnetic waves in $\mathrm{THz}$ region from highly optical nonlinear materials.

\subsection{Results and Discussions}

\subsection{Second order nonlinearity THz generation:}

Mathematical equations 39 and 42 are simulated using Maple and Matlab softwares. Simulations (using maple) are shown in Fig. 2.These simulations are done using integral equations. Initially in simulation current density, $\mathrm{J}$ is assumed as unit magnitude. Incident electric field is shown in Fig. 2. a and 2.b.Incident wave $E^{0}$ is of $85 \mathrm{MHz}$. The 3D grid with $100 \times 100 \times 100$ cells in total simulation region is used. Discrete step size is $\Delta x, \Delta y, \Delta z=1 \mathrm{~nm} \Delta x \Delta y \Delta z$ volume is considered for $\Delta V$.The radiation of $E 1$ is plotted using Maple in Fig. 2.c. and E2 is plotted in Fig. 2.d and e. Fig. 2.c depicts the 3-D plot of the first order perturbation to the electric field ie $E^{1}(\omega, r)$ while Fig. 2.d. displays the wave front 3D plot of the second order perturbation $E^{2}(\omega, r)$ to the electric field from basic perturbation theory applied to the Maxwell's equations. $E^{1}(\omega, r)$ is a linear functional of the tensor product of unperturbed electric field $E^{0}(\omega, r)$ while $E^{2}(\omega, r)$ is a linear functional of the tensor product of $E^{0}(\omega, r)$ represented by $E^{0}(\omega, r) \otimes E^{0}(\omega, r)$ so $E^{2}(\omega, r)$ contains coupled frequencies of $E^{0}$ ie $\omega_{0} \pm \omega_{1}$ where $\omega_{0}, \omega_{1}$ are dominant frequencies of $E^{(0)}$ as a result $E^{2}$ plot will contain more packed wave fronts.

Again Using Matlab the results are obtained for the susceptibility matrix of noncentrosymmetric /centrosymmetric materials defined in 1, the simulation works for the second order and third order harmonics ${ }^{30}$.The output is simulated for the frequency inputs $100 \mathrm{rad} / \mathrm{sec}, 10^{4}$ $\mathrm{rad} / \mathrm{sec}, 10^{5} \mathrm{rad} / \mathrm{sec}$ and $10^{12} \mathrm{rad} / \mathrm{sec}$. The $\mathrm{psd}$ is found highest at the input frequency of $10^{5} \mathrm{rad} / \mathrm{sec}$ and $10^{12}$ $\mathrm{rad} / \mathrm{sec}$. Here in this experiment plots are using a

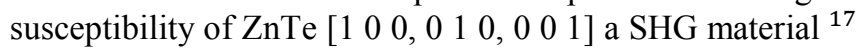
.This has been proved a material working at $\mathrm{THz}$ frequency from 0.5 to $10 \mathrm{THz}{ }^{25}$. The results vary if workspace matrix is a different matrix than the noncentrosymmetric susceptibility matrix. The autocorrelation function of perturbed wave is shown in Fig. 3. a and 3. b. Power spectral density (psd)/ periodogram using ZnTe (noncentrosymmetric material) is shown in Fig. 4. a.
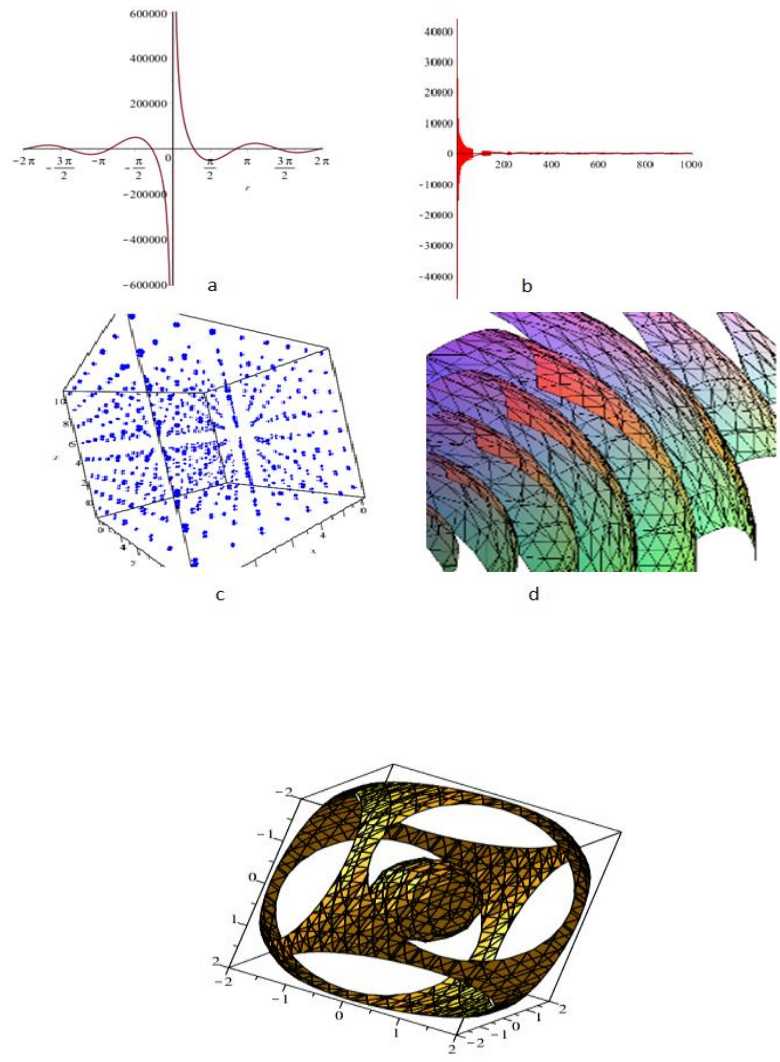

(e)

Figure 2: Input wave and simulated scattered wave plots using Maple: (a) Real part of incident wave $E^{0}$ (b) variation of incident electric field $E^{0}$ with $\mathrm{r}$ in nm (c) Scattered EM field (simulation of equation 39) (d) Wavefront of the scattered signal due to perturbed electric field $E^{2}(\mathrm{THz})$ (simulation of equation 42) (e) $3 \mathrm{D}$ plot of $E^{2}$

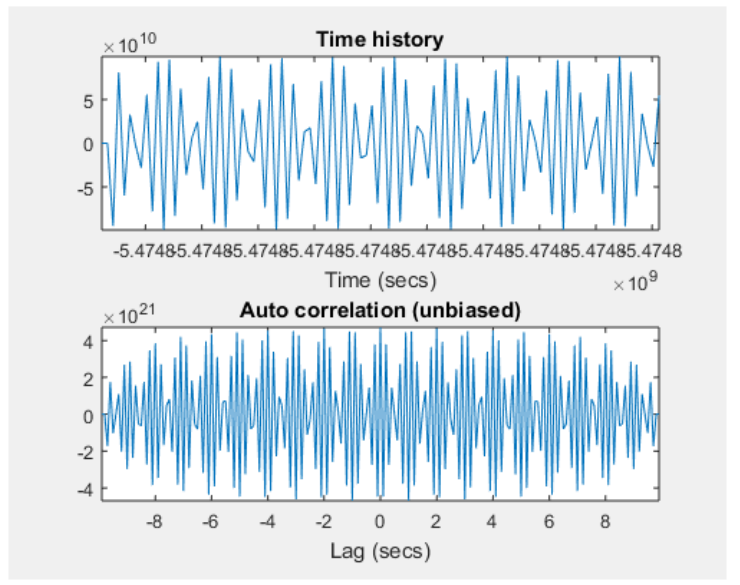

Figure 3: (a) First order perturbed wave autocorrelation, (b) Output SHG wave autocorrelation using Matlab 


\subsection{Third order nonlinearity and THz generation :}

The simulation results of equation 50 are obtained for the third order nonlinearity. Initially we assume a cavity of a nonlinear material/photonic crystal of $\Delta x \Delta y \Delta z$ and a femtosecond wave is incident on it. The output nonlinear wave equation shows first order perturbation and second order perturbations in Fig. 5. Periodogram for an isotropic case for $3^{\text {rd }}$ order nonlinearity is shown in Fig. 4.b. The interaction between scalar and vector wave components of the nonlinear wave equation in section 2.2 is obtained by correlation between them. Taking their FFT results in a constant envelope change resulting in a new sine wave. The output of these nonlinear wave equations using equation 42 with SHG and THG materials susceptibilities also shows a multipeak spectrum in $\mathrm{THz}$, the psd (power spectral density) plots shown in Fig. 4.a. and 4.b.Here different spectral peaks have different states of polarization ${ }^{16}$. These material susceptibilities are tested by creating a pattern of isotropic and anisotropic matrix in 2 dimensional space using Simulink model in Matlab. Fig. 6. also shows a psd plot of the third order nonlinear wave when simulated in Matlab code. High peak of psd is shown in lower THz.

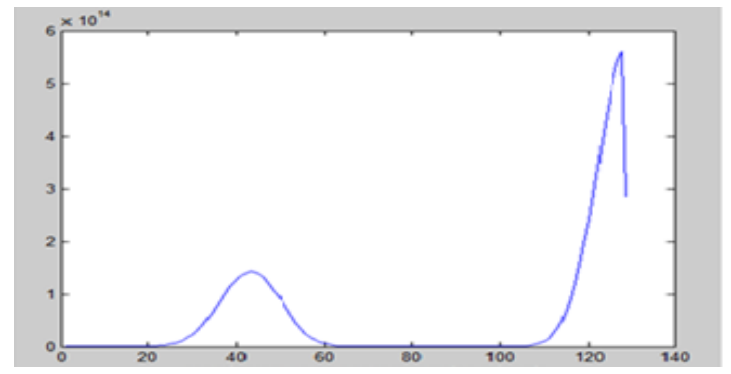

(a)

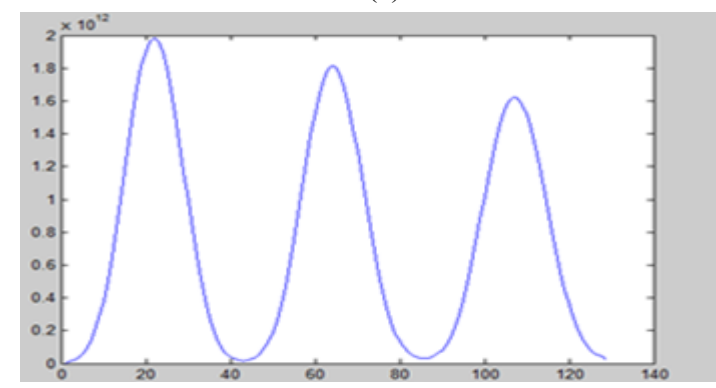

(b)

Figure 4: (a) Periodogram of output SHG wave using Welch in THz radiation, (b) Periodogram of output THG wave using centrosymmetric material

\subsection{Soliton generation:}

The FFT of the cross correlation between the scalar and vector output wave components of equation 50 shows interaction between two nonlinear waves which results in a sine wave spectrum propagating through the medium. Fig. 7.b. shows the waves propagating in the picosecond rate.

Variation of output wave is from - 1 to 0.This shows that these waves can propagate at low voltages, which can be propagated and received with miniature circuits. Rottwitt, etal. in 2014 see ref 16 proved the generation of solitons inside the medium which shows peaks of psd of waves in $\mathrm{THz}$ region.

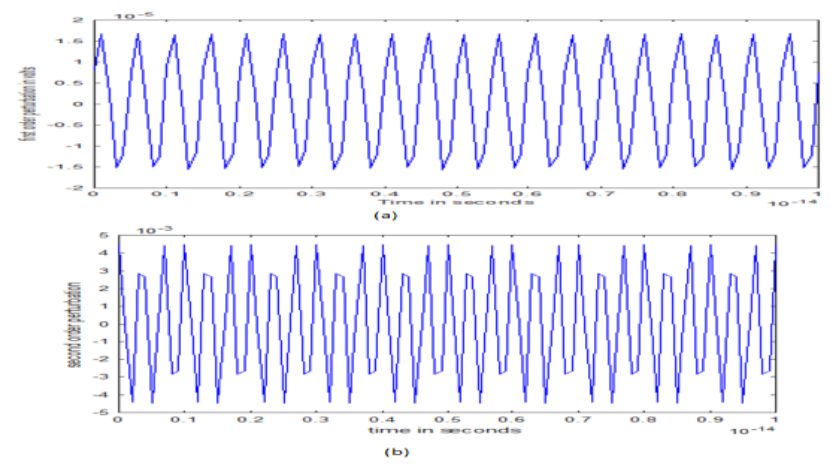

Figure 5: (a) First order perturbation, (b) second order perturbation using Kerr nonlinearity

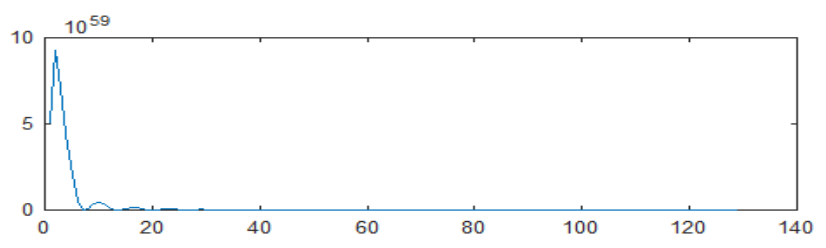

Figure 6: psd of output in $\mathrm{THz}$ range due to Third Harmonic wave

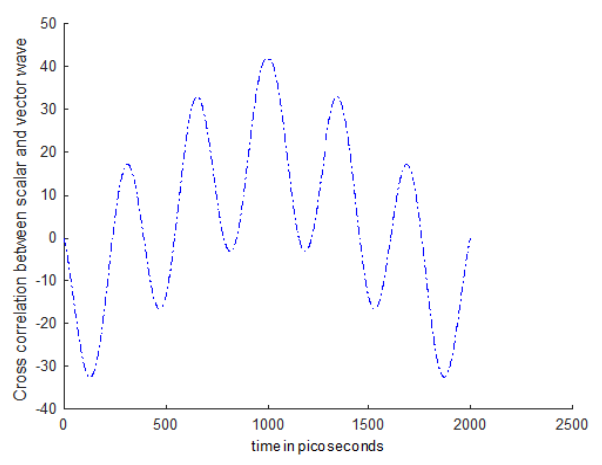

(a)

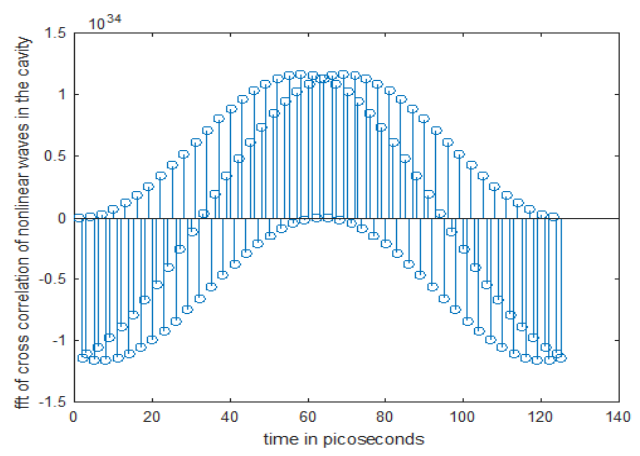

(b)

Figure 7: (a) Correlation between scalar and vector wave components, (b) FFT plot of the wave 
4.4. Wide Bandwidth: By solving the nonlinear wave equations of a third, order nonlinearity the output spectrum

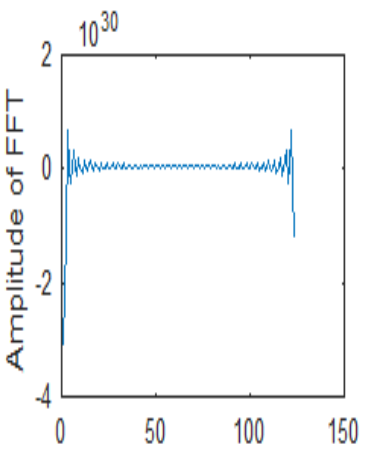

(a) frequency in $\mathrm{THz}$

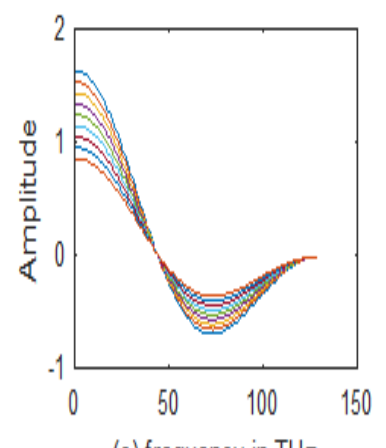

(c) frequency in $\mathrm{THz}$
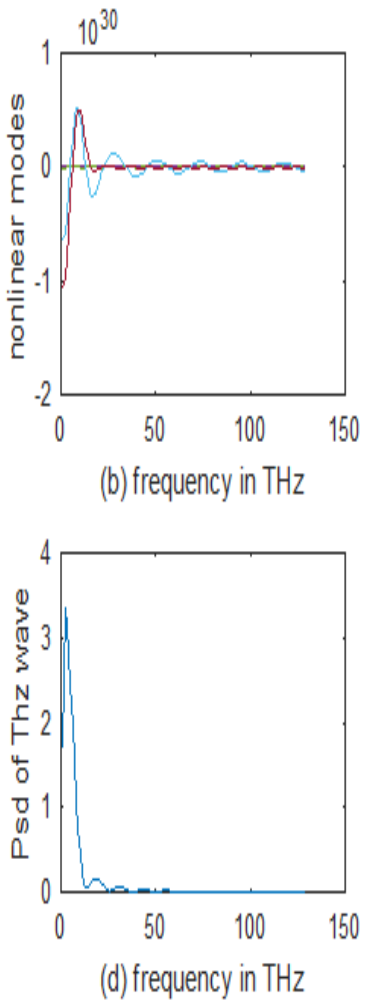

Figure 8: Outputs of a nonlinear wave obtained by a Kerr nonlinear material showing (a) Super continuum effect (b) modes in a nonlinear waveguide (c) Spectrum of waves in time frequency domain (d) Psd of a $\mathrm{THz}$ wave

is generated. FFT of the output THG wave shows a wide spectrum in Fig. 8. It gives different modes existing in the nonlinear wave. In addition, a sharp peak output power is achieved in low $\mathrm{THz}$ range. $\mathrm{THz}$ antennas are in high demand in High bandwidth communication. FFT of nonlinear wave output shows a broad pulse. All of these nonlinear processes are capable of generating new frequencies outside the input pulse spectrum. For sufficiently intense pulses, the pulse spectrum can become so broad that it extends over a frequency range exceeding $100 \mathrm{THz}$. It is also called super continuum ${ }^{31}$ which is also relatively flat over the entire bandwidth ${ }^{32}$.It is proved in the experiment that using these coupling of nonlinear waves we get this super continuum effect. In this simulation, we achieve a wide spectrum of pulse in the range of $100 \mathrm{THz}$ (Fig. 8.a.).

4.5. Number of Modes and spectrogram: Nonlinear effects in a waveguide can be used to make many active and passive components. Third order nonlinearity uses self-phase modulation(SPM) which is used in soliton formation and super continuum generation ${ }^{32}$. The simulation of nonlinear wave in Fig. 8.b. shows many modes propagating in the medium. The spectrogram in Fig. 8.c. shows the slowly time varying spectrum in the amplitude at each frequency. It uses the Short Time Fourier Transform $\int_{-\infty}^{+\infty}<X(t+\tau / 2) X(t-$ $\tau / 2>e^{j \omega \tau} \mathrm{d} \tau$. With two terms there is slow variation in the amplitude spectrum at each frequency.

4.6. THz Antennas: Second harmonic generation from a nonlinear material and $\mathrm{THz}$ generation from nonlinear patch is discussed in section 3. The antenna is designed with GaAs substrate and $\mathrm{T}$ shape patch at the four corners working as $\mathrm{T}$ patch resonator. (Fig. 9.a.) and (Fig. 9.b.).

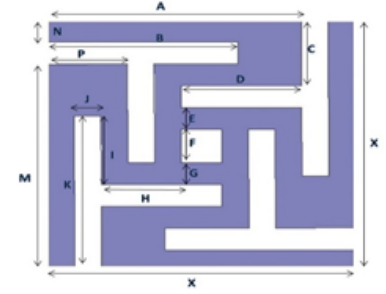

(a)

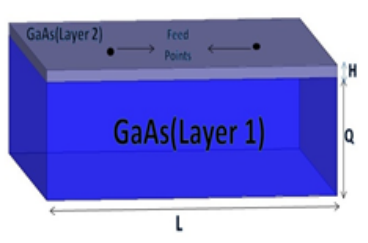

(b)

\begin{tabular}{|l|l|l|l|l|l|l|}
\hline Parameter & X & N & M & K & P & \\
\hline Value (mm) & 0.46 & 0.04 & 0.38 & 0.285 & 0.12 & \\
Parameter & J & A & B & I & L & \\
\hline Value (mm) & 0.04 & 0.38 & 0.285 & 0.125 & 0.12 & \\
\hline Parameter & G & D & C & E & F & \\
\hline Value (mm) & 0.04 & 0.18 & 0.12 & 0.04 & 0.1 & \\
\hline Parameter & Q & H & & & & \\
\hline Value (mm) & 0.125 & 0.013 & & & & \\
\hline
\end{tabular}

(c)

Figure 9: THz antenna design (a)Top view of patch antenna with $\mathrm{T}$ shape patches at the four corners (b) side view of patch antenna showing GaAs substrate (c) antenna dimensions

The perturbation of electric and magnetic field inside the medium and their interaction causes higher order fields that result in the electromagnetic scattering of waves. Fig. 9.c. shows the dimensions of the patch. The sum frequency generation inside the medium causes $\mathrm{THz}$ generation. The reflection coefficient s11 is shown in Fig. 9.d gives cut off frequency at $524.8 \mathrm{GHz}$ and high bandwidth of $25 \mathrm{GHz}$. Fig. 9.e. shows the gain of the antenna.

Third harmonic waves are produced by the Kerr nonlinearity. We derived the mathematical equations for the susceptibility which changes with electric and magnetic fields, with applied input $\mathrm{EM}$ wave. $\mathrm{THz}$ antenna using third order nonlinearity using Graphene patch with $\mathrm{SiO} 2$ substrate is also simulated .The results are shown in Fig 10 a-d. Fig.10.a. shows the design of bow tie antenna. Dimensions are shown in Fig. 10.b. The wave input is given by the coaxial cable. S11 is obtained at the frequency $3.5 \mathrm{THz}$ (Fig. 10. c.). The antenna gives a very high bandwidth of $2.5 \mathrm{THz}$. The electric field radiation pattern Ez and the gain of the antenna are shown in Fig. 10. d and $10 \mathrm{e}$.

\subsection{Bandwidth sensitivity to antenna material and geometry:}

GaAs and $\mathrm{SiO} 2$ both are semiconductor materials, which gives large nonlinear optical response [6]. GaAs is known to 


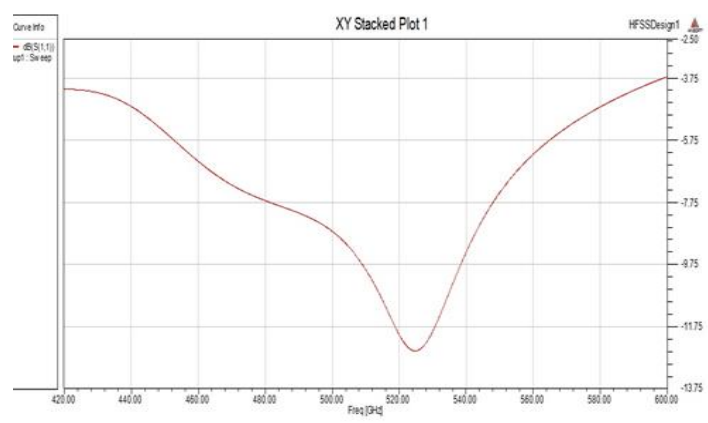

(d)

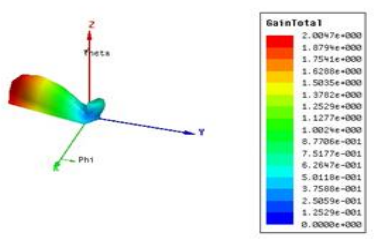

(e)

Figure 9: (d) S11 plot of radiation at $524.8 \mathrm{GHz}$, bandwidth $25 \mathrm{GHz}$, (e) Gain of Antenna

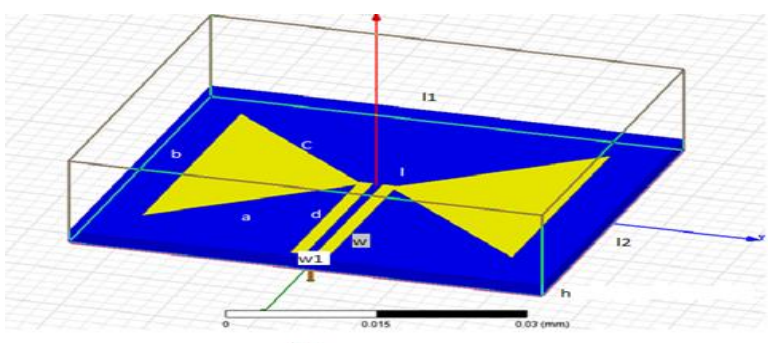

(a)

\begin{tabular}{l|l|l|l|ll|}
\hline Parameter & $\mathrm{a}$ & $\mathrm{b}$ & $\mathrm{c}$ & $\mathrm{d}$ & 1 \\
\hline Value $(\mathrm{mm})$ & 0.02315 & .0312 & .02315 & .02087 & .0218 \\
\hline Parameter & $\mathrm{w}$ & $\mathrm{w} 1$ & 11 & 12 & $\mathrm{~h}$ \\
\hline Value $(\mathrm{mm})$ & .0015 & .0010 & .0050 & .045 & .002 \\
\hline
\end{tabular}

$(b)^{-1}$

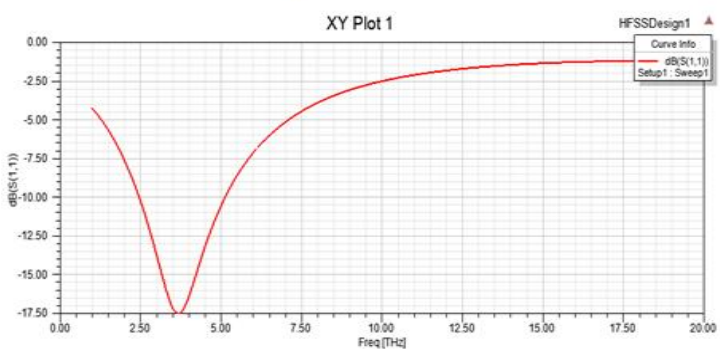

(c)

Figure 10: (a)Bow tie Graphene antenna on a $\mathrm{SiO} 2$ substrate,(b) dimensions in $\mathrm{mm}$, (c)s11 parameter

have second order nonlinear properties as regards polarization Vs electric field graph while Graphene patch with $\mathrm{Si} \mathrm{O}_{2}$ substrate is known to have third order nonlinear properties as regard to polarization Vs electric field graph. Therefore, in the analysis of the former second order perturbation theory is
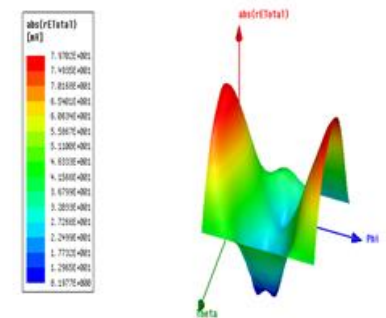

(d)
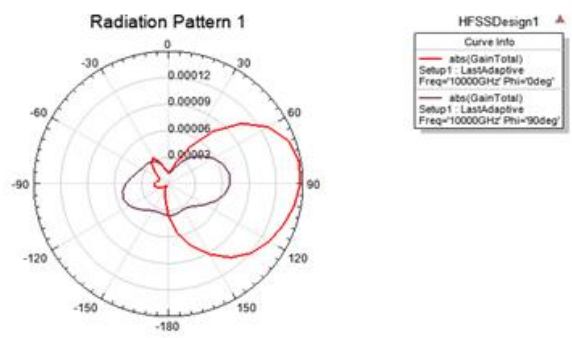

(e)

Figure 10: (d) Radiation pattern, (f) Gain total of a single bow tie graphene antenna over $\mathrm{SiO} 2$ Substrate

needed whereas in that of the later third order perturbation theorem calculations are required in order to get sufficiently good approximations for the radiated field. Second harmonic generation converts almost all input incident wave to radiation [6].It is a quantum mechanical process where two photons of $\omega$ are destroyed and a photon of $2 \omega$ is simultaneously created. Moreover in the former case $\omega_{1} \pm$ $\omega_{2}$ will be the dominant frequency of the radiated field and the presence of radiation at frequency $\omega_{1} \pm \omega_{2}$ stimulates the emission of additional photon at these frequencies in the infrared wavelengths [6].In case of third order nonlinear optical process latter $\omega_{1} \pm \omega_{2} \pm \omega_{3}$ will be the dominant frequencies where $\omega_{1}, \omega_{2}, \omega_{3}$ will be the dominant frequencies in the incident electric field [6]. Since $\omega_{1} \pm \omega_{2} \pm$ $\omega_{3} \geq \omega_{1} \pm \omega_{2}$ hence antenna, using latter material will have higher bandwidth.

Antenna geometry also plays an important role here. In an experiment using graphene as a rectangular patch with $\mathrm{SiO} 2$ substrate, ${ }^{35}$ it shows strong absorption in the $5.2 \mathrm{THz}$ and $8.5 \mathrm{THz}$ and with a bow tie geometry. Graphene patch with $\mathrm{SiO} 2$ substrate shows $\mathrm{S} 11$ at $3.5 \mathrm{THz}$ with bandwidth $2.5 \mathrm{THz}$. Again, if we change the material of substrate with same bow tie geometry we have found the following changes ${ }^{35}$ :

4.7.1. With Si substrate it shows dual bands at $12.55 \mathrm{THz}$ and $13.65 \mathrm{THz}$ with huge bandwidth $12.15 \mathrm{THz}-13.0 \mathrm{THz}$ and $13.6 \mathrm{THz}-13.8 \mathrm{THz}$.

4.7.2. With $\mathrm{Al} 2 \mathrm{O} 3$ substrate of same width, it gives multiple bands at $18.05 \mathrm{THz}, 23 \mathrm{THz}, 20.25 \mathrm{THz}$, and $21.5 \mathrm{THz}^{35}$. 
The antenna also shows Fabry Perot resonator like resonance giving multiple bands from 1.5 to $18 \mathrm{THz}$ when we change the sweep frequency. $\mathrm{Al}_{2} \mathrm{O}_{3}$ is a third order nonlinear material which is also a semiconductor material.

4.7.3. Graphene with $\mathrm{SiO} 2 / \mathrm{Si}$ substrate and with Gold deposition shows radiation in $\mathrm{THz}$ range ${ }^{36}$ at $120 \mathrm{GHz}$.

Hence, High-speed data transmission using large bandwidth is possible with nanoscale using some newly found materials such as Graphene, Carbon Nano tubes ,Gold and Silver ${ }^{37}$. Hence, materials can control the bandwidth as well as radiation in the $\mathrm{THz}$ region.

As we discussed that both antenna material and geometry controls the Antenna bandwidth. Antenna geometry has dominant role to control the bandwidth as spiral antennas show variation in radiation and bandwidth with single aperture, which is independent of frequency [49]. On the other hand, if the material is for example a $3^{\text {rd }}$ order nonlinear material it may contain $\omega_{1}+\omega_{2}+\omega_{3}$ as dominant frequencies but not $\omega_{1}, \omega_{2}, \omega_{3}$ individually. Third order nonlinear material ( $\mathrm{SiO}_{2}$ and $\left.\mathrm{Al}_{2} \mathrm{O}_{3}\right){ }^{38,39}$ with Graphene shows increase in bandwidth as compared with the substrate of second order nonlinear material(GaAs). Graphene with $\mathrm{Si}$ waveguide has also achieved supercontinum effect with pulse compression which is very important to boost the speed and capacity of the future networks ${ }^{40}$.

Hence, choosing appropriate geometry of the SHG and Kerr nonlinear material one can design a micro strip Nano patch antenna for $\mathrm{THz}$ radiation depending on its applications.

\subsection{Comparison of modelling Graphene antenna with normal bulk material antenna:}

The design in the normal bulk material and the microwave region shows intrinsic impedance losses ${ }^{41}$.Physics of bulk material depends on the carrier dynamics for example $\mathrm{Fe}$ doped in bulk GaInAs ${ }^{42}$.Carrier lifetime should be ultrashort in these materials for possible photoconductive switching. It is found that Fe doped GaInAs is highly resistive and has sub picoseconds carrier lifetime ${ }^{42}$. Permittivity and permeability can be controlled by static $\mathrm{E}$ and $\mathrm{H}$ field consecutively in Ferroelectric bulk material ${ }^{43}$. It gives polarization configurability. Drawback of using Ferroelectric bulk material is that at the thickness of millimeters their conductivity becomes very high which can also degrade the performance of the antenna. Antenna configurability is useful in more complex controls. However full exploitation of antenna functionality may require dedicated signal processing and it has more complicated fabrication procedures as compared to traditional antennas ${ }^{43}$.

Antenna dynamic configuration is possible by using miniaturization in graphene ${ }^{38}$.Properly sized graphene in nanoscale gives $\mathrm{THz}$ antennas ${ }^{44}$, which has strong nonlinear optical properties. It has third order nonlinearity and four wave mixing property, which can be used for imaging. These antennas can be controlled by static electric and magnetic fields ${ }^{45}$. Graphene gives SPP (Surface Plasmon Polaritron) waves at terahertz band and in addition can be tuned by means of doping some materials ${ }^{46}$.SPP waves are excited at the interface between graphene and dielectric material. Properties of the surface waves ${ }^{47}$ can be controlled by tailoring the geometrical parameters of these antennas. Graphene also has mechanical flexibility .Its conductivity is the main property. TM surface Plasmon are created at the two dielectric layer interface due to which there is variation in the conductivity ${ }^{48}$. Conductivity of Graphene changes from DC to Terahertz band up to visible band. Hence, graphene based Nano antennas in micrometer size are able to work for Nano networks at Terahertz bands.

\subsection{Applications:}

Materials with excellent photo physical properties and large optical nonlinearities have many potential applications including fast optical communication, all optical switching; optical limiting ${ }^{7}$.Other than this some major applications of nonlinear optics are given below:

6.1. Inverse scattering: Using nonlinear wave optics, we can extract the parameters of an unknown object or a medium ${ }^{15,34,34}$.The techniques are used in inverse scattering. 6.2. Optical Nano switch: Second order nonlinearity can be used as a Nano switch. Nano switch is made of nonlinear crystal, where a pump wave controls the output wave. These devices are used in active devices and gives wide scope for low power applications 27 .

6.3. RF Imaging: $\mathrm{THz}$ waves produced by optical nonlinearities are of the wavelength of a molecular size.It is penetrable to $\mathrm{mm}$ to micrometer wavelengths and non ionizing hence can be used to detect buried objects, hidden objects.In these equipments radiator transmits waves by passing through a nonlinear medium and detector detects that medium which is the main cause of its generation hence can be used as a sensor. By using signal processing techniques 18 ,image is enhanced and details are obtained. Therefore in Military application, security imaging and in secured data communication $\mathrm{THz}$ technology has to play significant role and it has potential for imaging, communication and sensors.

6.4. Application in clinical and preclinical research: As the $\mathrm{THz}$ waves are nonionizing unlike X-ray imaging so not causing any damage to the tissues. Certain biological materials as discussed in one are highly polarizable, as these materials are assembled from fairly ordered, large noncentrosymmetric structures ${ }^{2,23}$. Alteration of biological materials in a diseased state affects the level of SHG obtained from the imaged tissue, making it a useful optical property for diagnostic purposes by nonlinear optical materials 2,23 . Promising application are in the inspection of biomedical tissue, separation of tumor cells from normal tissues, threedimensional view of tooth decay ${ }^{30}$. Therefore in medical imaging it has now become possible to design sensors in which we can detect a defect or foreign cells in the biological tissue.

\subsection{Conclusion}

Here in this paper we derived set of equations for emission of SHG and THG waves from an optical nonlinear material .The simulations were performed for isotropic and aniosotropic materials which gives $\mathrm{THz}$ radiation. These SHG and THG waves which are obtained by nonlinear materials are the main source of $\mathrm{THz}$ waves. Parameters are also obtained using 
matlab code and HFSS model using Finite element method. In the Antenna design we use a nonlinear substrate of GaAs which proves $\mathrm{THz}$ radiation from second order nonlinearity. Graphene antenna with $\mathrm{SiO} 2$ substrate which falls in the third order nonlinear materials gives results in $\mathrm{THz}$ range also with wide bandwidth in THz.Hence taking different geometry of these antennas based on SHG and THG materials different Terahertz antennas can be designed for different applications.As compared to the normal bulk antennas these antennas are easily tunable in micrometer length in terms of radiation and bandwidth. These antennas also give solutions to the inverse scattering and imaging techniques.

\section{References}

[1] M.Khulbe, M.R.Tripathy, H.Parthasarathy, Wavelet based method for nonlinear Inverse scattering problem using least mean square estimation" Progress In Electromagnetic Research Symposium, St. Petersburg, Russia, 22-25 May (2017) online ieee explore

[2] A Y. Pawar, D. Sonawane, K.B. Erende, D. V. Derle, Terahertz Technology and its Applications, Elsevier Drug Invention Today, 5(2) (2013)157-163

[3] X. Zhang, C. Chung, S. Wang, H. Subbraman, Z. Pan, Q.Zhan, Integrated broadband bowtie antenna on transparent Silica Substrate, IEEE Antennas and Wireless Propagation Letters, (2016) 1-4

[4] B. Zhu, Y. Chen, K. Deng, W.Hu, Z.S. Yao ,Terahertz science and technology and applications, PIER Proceedings, Beijing China(2009) March 23-29

[5] Summit High Frequency Electronics, Terahertz (THz) Technology: An Introduction and Research Update, Technology Report, Summit Technical media LLC, (2008) 40

[6] R. B. Boyd, Nonlinear Optics, (Elsevier, $3^{\text {rd }}$ edition.2008)5-10, 69-70,240

[7] L. ZhiBo, Z. XiaoLiang, Y. XiaoQing, C. YongSheng \& T. JianGuo, Nonlinear optical properties of graphene-based materials ,Chinese Science Bulletin, Review, Special Issue Graphene, vol. 57,no-23, Aug( 2012) 2971-2982

[8] J. A. Zielinska and M.W. Mitchell, The photo-Kerr effect: a new optical nonlinearity and its use for cavity selfstabilization, Letters, Optica, vol. X, No. X July 31, 2017

[9] I. G. Koprinkov, A. Suda, P. Wang, and K.Midorikawa, Self-Compression of High-Intensity Femtosecond Optical Pulses and Spatiotemporal Soliton Generation, Phys. Rev. Lett. 84, (2000) 3847

[10] Y.Guo, C.K. Kao,E.H. Li , K.S. Chiang, Nonlinear Photonics,( Springer 2002)18, 21-29
[11] D. J. Hagan, and P.Kick, G. Kik, Light Matter Interaction, Lecture notes, CREOL, The college of Optics and Photonics, Florida (2013)

[12] A.Balanis, Antenna Theory and Design, (Wiley Pub, $2^{\text {nd }}$ Edition 2015)118,120,125

[13] J. Kroha, C. M. Soukoulis, P. Wolfie, Localization of classical waves in a random medium: A self-consistent theory, Physical Review B, American Physical Society, vol. 47, no 17 (1993)

[14] H., Harutyunyan, V. Giorgio, and L., Novonty, Nonlinear Optical Antenna, (Cambridge University Press 2013),

[15] D.Sjöoberg, Direct and Inverse Scattering of Electromagnetic Waves in Nonlinear Media, Department of Electromagnetic Theory, Lund Institute of Technology, PhD thesis, (1999) 4-16

[16] K Rottwitt, P Tidemand-Lichtenberg, Nonlinear Optics: Principles and Applications, (CRC Press, 2014)

[17] Peter H. Wagner, M. Kuhnelt, Langbein, W. Wolfgang, J. M. Hvam, Dispersion of the second order nonlinear susceptibility in ZnTe, ZnSe and ZnS, Physical Review B (Condensed matter and material physics), vol. 58 no-16 oct 15 (1998) 494-497

[18] M.Khulbe, H. Parthasarathy, Soliton generation in a Kerr medium: mathematical analysis using Maxwell's equations, International Journal of Photonics (IJP), vol 5,no 1-2,pp1-12

[19]M.Khulbe,H.Parthasarathy,M.R.Tripathy, Mathematical analysis of RF Imaging techniques and Signal processing using wavelet transforms, International Journal of Signal and Imaging System Engineering, Inderscience Journal V.10 N6 (2017)

[20]L. Kavitha, E. Parasuraman, D. Gopi \& S. Bhuvaneswari, Propagation of electromagnetic solitons in an antiferromagnetic spinladder medium, Journal of Electromagnetic Waves and Applications, Vol. 30, Issue $6(2016)$ 740-766

[21] A Passively Mode-Locked Erbium-Doped Fiber Laser Based on a Single-Wall Carbon Nanotube Polymer F. Ahmad, S. W. Harun ${ }^{1,3}$, R. M. Nor ${ }^{4}$, N. R. Zulkepely ${ }^{4}$, H. $\mathrm{Ahmad}^{3}$ and P. Shum ${ }^{5}$, Chinese Physical Society and IOP Publishing Ltd Chinese Physics Letters, Volume 30, Number 5(2013)

[22] C.L. Tang, Nonlinear and Photorefractive Optics, Nonlinear Optics, (McGraw-Hill, Inc. ch-38, 1995) 19-20 
[23] G.Thomas, J. V.Voskuilen , H. C. Gerritsen, H.J.C.M. Sterenborg, Advances and challenges in label-free nonlinear optical imaging using two-photon excitation fluorescence and second harmonic generation for cancer research, $J$ Photochemical Photobiology B Dec 141(2014)128-38.

[24] R.K. Chang, J. Ducuing and N. Blomberger, Relative phase measurement between fundamental and second harmonic light, Phys. Rev .Lett ,15 (1965) 415

[25] J.L. Cheng, N. Vermeulen, J.E Sipe, Second order optical nonlinearity of graphene due to electric quadrupole and magnetic dipole effects, Scientific Reports, March 6(2017) 1-2

[26] J. Danielson, Generation of narrowband $\mathrm{THz}$ pulses and $\mathrm{THz}$ studies of ultrafast phenomenon, Thesis Feb, Oregon State University, USA (2008)

[27] S. Farazi, and A. Zarifkar., A Low-Power Optical Nano switch Based on XPM-Enhanced Second Harmonic Generation, Journal of Light wave Technology, Vol. 35, Issue 10(2017) 1988-1994

[28] N.A. Savostianova, and S.A. Mikhailov, Third harmonic generation from Graphene lying on different substrates: Optical phonon resonances and interference effects, Optics Express 25 (2017) 8-9

[29] R.I. Woodword, R.T. Murray, C.F. Phelan, R.E.P Oliveira, T.H. Runcorn, E.J.R.Kelleher, S.Li3, E.C. Oliveira, G.J.M. Fechine, G. Eda and C J S Matos, Characterization of the second and third order nonlinear optical susceptibilities of monolayer MoS2 using multiphoton microscopy, 2D materials, letter, IOP,(2017),4

[30] X. Yin, B.W.H Ng, D. Abbott ,Terahertz imaging for biomedical applications pattern recognition and tomographic reconstruction, Springer Science \& Business Media (2012), 6

[31] Q. Lin and G.P. Agrawal, Vector Theory of Cross-Phase Modulation: Role of Nonlinear Polarization Rotation, IEEE of Journal of Quantum Electronics Vol. 40, no. 7, July (2004)

[32] JK Ranka, RS Windeler, AJ Stentz, Visible continuum generation in air-silica microstructure optical fibers with anomalous dispersion at $800 \mathrm{~nm}$, Optics letters, Vol. 25, Issue $1,(2000)$, pp. $25-27$

[33] Q. Lin, O. J. Painter, G. P. Agrawal, Nonlinear optical phenomena in silicon waveguides, Opt. Express 15, (2007), 16604

[34] G. Kristen son and D. J N Wall ,Direct and inverse scattering for transient electromagnetic waves in nonlinear media, IOP, Inverse Problems, Volume 14, No 1(1997)
[35] M.Khulbe, M.R.Tripathy H. Parthasarathy, "Plasmonics for $\mathrm{THz}$ applications: Design of Graphene square patch antenna tested with different substrates for $\mathrm{THz}$ Applications", Next Generation Computing Technologies, Springer conference ,30-31Oct2017,Dehradun,India, Proceedings book chapter of "Communications in Computer and Information Science (CCIS) "Series ISSN No: 18650929,vol 747 (under press)

[36]. R.Bala, A. Marwaha, "Characterization of graphene for performance enhancement of patch antenna in THz region", Elsevier (2014)

[37] A.S. Thampy, M.S. Darak,S.K. Dhamodharan, "Analysis of graphene based optically transparent patch antenna for terahertz communications, Journal of Physics, Physica E: Low-dimensional Systems and Nanostructures", vol. $66,(2015)$

[38] H.Elayan, R.M.Shubair, A.Kiourti, "On Graphene based THz plasmonics Nano Antenna", Microwave Symposium (MMS), 2016, 16th Mediterranean, Abu Dhabi, United Arab Emirates,

[39] M.Nadafan, Z. Dehghan, Rasoul Malekfar,Microstructural and nonlinear optical properties of $\mathrm{SiO} 2$ and $\mathrm{Al} 2 \mathrm{O} 3$ nanoparticles doped in polyurethane,Vol. 30, Issue 11,14 June 2015, pp. 17881796

[40] A. Ishizawa, R. Kou, T. Goto,T. Tsuchizawa, N. Matsuda, K.Hitachi,T.Nishikawa, K.Yamada, T.Sogawa, and H. Gotoh, "Optical nonlinearity enhancement with graphenedecorated silicon waveguides", Science Rep. 2017; 7: 45520.Published online 2017 Apr 12.

[41]J.Paruisseaee, Tamagnone, C. M., Gomez- Diaz J.S., Carrasco, E. "Grapheme Antennas; Can Integration and Configurability Compensate for Loss", Microwave Conference (EuMC), (2013)

[42] S. Sengupta, "Characterization Terahertz Emission from High resistivity $\mathrm{Fe}$ doped Bulk $\mathrm{Ga}_{.69} \mathrm{In}_{.31} \mathrm{As}$ based photoconducting antennas" Doctoral Thesis ,Springer , Renesselaer Polytechnic Institute,Troy,USA,2011 page 47

[43] J. T. Bernhard, "Reconfigurable antennas" (Synthesis lectures on Antennas), Morgan \& Clypool Publisher, pg. 19, 20

[44]I. Llatser, C. Kremers, Cabellos-Aparicio, A., Jornet, J.M., Alarcon, E. and Chigrin, D.N.: Graphene-based Nanopatch antenna for terahertz radiation, Photonics and Nanostructures fundamentals and application, 2012 vol.10, pp.353- 358 
[45] J.Paruisseaee, C. M. Tamagnone, Gomez- Diaz J.S., Carrasco, E.: Grapheme Antennas; Can Integration and Reconfigurability Compensate for Loss, Microwave Conference (EuMC), 2013

[46] J. M. Jornet and I. F. Akyildiz "Graphene-based Plasmonic Nano Antenna for Terahertz Band Communication in Nano networks", IEEE Journal on Selected Areas in Communications/Supplement-Part2,Vol.31,No.12, Dec 2013

[47] M. Rahm, Tahsin Akalin, Ajay Nahata, Miguel

Beruete , "Focus on Terahertz plasmonics" 2015, New J.

Phys. 17

[48] I. F. Akylidiz, M. Jornet: Graphene based Plasmonic Nano antenna for terahertz, IEEE Journal on Selected Areas in Communication, vol. 1, Elsevier Science (2002)

[49] D.S. Filipovic, J. L. Volakis slot spiral antenna designs for dual-band/multiband operation, IEEE Transactions on Antennas, Vol. 51 Issue: 3430 - 440, 28 May 2003

[50] K. Imakita, M. Ito, M. Fujii, and S. Hayashi, "Nonlinear optical properties of $\mathrm{Si}$ nanocrystals embedded in $\mathrm{SiO} 2$ prepared by a co sputtering method", Journal of Applied Physics 105, 093531 (2009);

CTRL-F and try to find a common word such as 'the'). The proceedings editors will contact authors of non-complying files to obtain a replacement. 